\title{
Precision studies of vector-boson production with heavy quarks at the LHC: the case of $Z+b$ jet.
}

\section{Figueroa, ${ }^{a}$ S. Honeywell, ${ }^{a}$ S. Quackenbush, ${ }^{a}$ L. Reina ${ }^{*}{ }^{a}$ C. Reuschle ${ }^{a}$ and D. Wackeroth ${ }^{b}$}

${ }^{a}$ Physics Department, Florida State University,

Tallahassee, FL 32306-4350, U.S.A.

${ }^{b}$ Department of Physics, SUNY at Buffalo,

Buffalo, NY 14260-1500, U.S.A.

E-mail: daf14f@my. fsu. edu, s jh07@hep. fsu . edu,

squackenbush@hep.fsu.edu, reina@hep.fsu.edu, creuschle@hep.fsu.edu,

dow@ubpheno.physics.buffalo.edu

\begin{abstract}
After a brief introduction to the state of the art of theoretical predictions for electroweak vectorboson production with both top and bottom quarks at the LHC, we review the case of Z-boson production with $b$ jets, and discuss the impact of NLO QCD+EW corrections and finite $b$-mass effects on the theoretical prediction for $Z+b$ jet production.
\end{abstract}

Loops and Legs in Quantum Field Theory (LL2018)

29 April 2018 - 04 May 2018

St. Goar, Germany

\footnotetext{
* Speaker.
} 


\section{Overview and Outlook}

The production of electroweak (EW) vector bosons $\left(V=W^{ \pm}, Z\right)$ with heavy quarks ( $b$ and $\left.t\right)$ is particularly relevant to the physics of the Large Hadron Collider (LHC) for several reasons.

First of all, the associated production of $W$ or $Z$ bosons with heavy quarks constitutes an important background for Higgs physics and searches of new physics beyond the Standard Model (SM). For example, $V+t \bar{t}$ with $V$ decaying into leptons is the dominant background for measuring the production of a SM Higgs boson in the $t \bar{t} H$ channel followed by $H \rightarrow W^{+} W^{-}$(with subsequent decay to $l^{+} v l^{-} \bar{v}$ or $l v q \bar{q}^{\prime}$ ), $H \rightarrow Z Z$ (with subsequent decay to $v \bar{v} q \bar{q}$ or $l^{+} l^{-} q \bar{q}$ ), or $H \rightarrow \tau^{+} \tau^{-}$. On the other hand, $W$ and $Z$ production with $b$ jets are the dominant backgrounds in the measurement of associated Higgs-boson production with either $W$ or $Z$, when the Higgs-boson decays into $b$ quarks $(H \rightarrow b \bar{b})$. Hence the accurate theoretical prediction of both $V+t \bar{t}$ and $V+b$ jets is a crucial element in testing the Higgs-boson couplings to third-generation quarks.

At the same time, both $V$ production with top and bottom quarks have their own intrinsic value in testing important aspects of weak and strong interactions. Measuring $V+t \bar{t}$ production provides a direct test of anomalies in top-quark electroweak couplings, while $W+t$ represents one of the components of single-top production, whose measurement could help constraining the Wtb coupling from single-top measurements. If $V+t \bar{t}$ and $V+t$ processes are specific domain of the LHC, given the massiveness of the particles produced, the analogous $V+b$-jet processes have been measured already at the Tevatron and sometimes left us with puzzling results that are now being revisited by the LHC. From the first round of LHC measurement we can see emerging a much more consistent picture, and we can start envisaging the possibility of using these processes as a testing ground to understand more complicated signatures involving $b$ jets (e.g. crucial background processes such as $t \bar{t}+b$ jets). Last but not least, increasing experimental precision and theoretical accuracy in the prediction of $V+b$-jet processes can provide a direct measurement of the $b$-quark parton distribution function (PDF), whose direct knowledge could constrain the theoretical parameterizations currently used in PDF fits.

Theoretical progress in providing more and more accurate predictions for both $V+t \bar{t}$ and $V+b$ jets has been steady over the last few years. The Next-to-Leading Order (NLO) QCD corrections to $Z+t \bar{t}$ and $W^{ \pm}+t \bar{t}$ production at the LHC have been calculated in [1,2] and [3] respectively, and interfaced with parton-shower in $[4,5]$ in the PowHel framework. The complete NLO QCD and electroweak (EW) cross sections for $t \bar{t}+V$ hadroproduction have more recently been presented in [6], and successfully compared with results from a calculation based on OpenLoops+SHERPA [7] in the context of the Higgs Cross Section Working Group [8]. In the same context [8], differential cross sections from existing fixed-order NLO QCD calculations of $V+t \bar{t}$ have been successfully cross checked. The theoretical uncertainty of the NLO QCD+EW $V+t \bar{t}$ total cross sections, due to both renormalization and factorization scale dependence, PDF, and $\alpha_{s}$, is estimated to be around 15-20\%, much improved if compared to the corresponding treelevel results. On the other hand, the cross sections for $W+t$ production has been calculated at NLO [9] and the improved cross section with resummation of next-to-next-to leading logarithms (NNLL) has been presented in [10], where the theoretical uncertainty is found to be around a few percents.

The case of $V+b$-jet hadroproduction has a more intriguing theoretical structure, due to both 
its intrinsic dependence on largely different scales $\left(m_{b}, M_{V}\right.$, and the characteristics energy of LHC collisions), which introduces the possibility of large logarithmic contributions in particular kinematic regions and energy regimes and prompt to their resummation, and to the possibility of also involving $b$-initiated processes. On top of refining the theoretical predictions by adding higher-orders of quantum corrections, theorists have also been debating the best approach to the calculation of the $V+b$-jet cross sections, with the aim of controlling and improving the stability of perturbative predictions. With this respect, at the NLO of QCD+EW corrections, the calculation of the hadronic production of $V+2 b$ jets can only proceed in strict analogy with $V+t \bar{t}$. On the other hand, the case of $V+1 b$ jet can be calculated assuming or not assuming an initial-state $b$ density, i.e. using a 5 -flavor scheme (5FS) or a 4-flavor scheme (4FS). In the first case (5FS) the definition of a $b$-quark PDF removes from the hard cross-sections some of the large logarithmic corrections due to initialstate radiation and resums them via renormalization-group scale evolution. In the second case (4FS) a more realistic description of hard final-state radiation can be achieved. Being the 4FS and 5FS just two different ways of reordering the perturbative expansion of the cross section, their predictions agree better and better the higher the perturbative order reached in a calculation. More extensive discussions of this issue has been presented in the literature [11, 12, 13, 14, 15, 16, 17, 18].

For $V+b$-jet processes, most calculations so far have focused on higher-order QCD corrections and interfacing fixed-order NLO QCD calculations with parton-shower event generators. In particular, NLO QCD corrections have been calculated for $W+2 j$ with at least one $b$ jet $[19,20,21]$, $W+2 b$ jets [22, 23, 24], $W+2 b$ jets plus a light jet [25, 26], $Z+1 b$ jet [27], $Z+2 j$ with at least one $b$ jet [28], $Z+2 b$ jets [29,23], where, depending on the process, either the 4FS or the 5FS have been used. The theoretical uncertainty of NLO QCD calculations, in particular for $W+b$ jets remains quite large, of the order of $20-25 \%$ or more, due to the opening of new large tree-level contributions among the real radiation processes. Recently, a very interesting study [30] that has pushed the NLO QCD calculation of $W+2 b$ jets plus $n$ light jets up to $n=3$ has been able to confirm that most residual scale uncertainty can indeed be cured by including higher-order corrections beyond NLO QCD. Although technically very challenging, this could be within reach in the close future. At the same time, realistic studies interfacing NLO QCD fixed-order calculations with parton-shower (PYTHIA [31, 32] and HERWIG [33, 34]) have been presented in the POWHEG BOX [35] framework for $W+2 b$ jets [36] as well as $W+2 b$ jets plus one light jet [25], and in the MaGraph5_aMC@NLO [37] framework for $W / Z+2 b$ jets [38]. A separate study using SHERPA+OpenLoops has appeared in [18]. All these studies represent the state of the art of theoretical predictions that have been and are compared with both Tevatron and LHC results (see [13] and [39] for a detailed discussion of these comparisons up to results from Run I of the LHC). Most of the fixed-order total and differential rates for $V+t \bar{t}$ and $V+b$ jets are available through MCFM [40].

Quite recently, combined NLO+EW correction to $Z$ plus one $b$-jet hadroproduction have been presented in [41], with attention to the effect of considering a massive $b$ quark both in the initial and final state. Results from this study will be presented in Section 2. It is crucial to notice that, in spite of their small size, controlling these effects is important. EW and $m_{b}$ effects can affect kinematic distributions in complementary regions, relevant to different measurements. Also, the case of initial-state massive partons [42] deserves particular attention in implementing a consistent matching between NLO fixed-order 5FS calculations and parton showers (see also [43]). 


\section{The case of $Z$ plus one $b$ jet: NLO QCD and EW corrections for a massive $b$}

The combined NLO QCD+EW total and differential cross sections for $Z+1 b$ jet have been calculated in [41] considering five flavors and the $b$ quark massive in both initial- and final-state processes, i.e. in the so called massive 5FS (or m5FS).

Results for the LHC with center of mass energy $13 \mathrm{TeV}$ have been presented in [41]. They have been obtained using the NLOX one-loop provider [44] for one-loop virtual QCD and EW corrections, and several in-house codes implementing both phase-space slicing and dipole subtraction for the real corrections. In order to take into account all $b$-mass effects, both the initial-state kinematics, the phase-space integration, the UV counterterms, and the PDF subtraction terms have been consistently modified. Full details can be found in [41].

Table 1 as well as Figs. 1 and 2 summarize the results of Ref. [41] by showing both the impact of NLO EW corrections and of initial-state $b$-quark mass effects on total and differential cross sections. NLO QCD and EW corrections are combined in both the additive approach,

$$
\sigma_{\mathrm{NLO}}^{\mathrm{QCD}+\mathrm{EW}} \equiv \sigma_{\mathrm{LO}}+\alpha_{s}^{2} \alpha \sigma^{(2,1)}+\alpha_{s} \alpha^{2} \sigma^{(1,2)}
$$

and the multiplicative approach

$$
\sigma_{\mathrm{NLO}}^{\mathrm{QCD} \times \mathrm{EW}} \equiv \sigma_{\mathrm{NLO}}^{\mathrm{QCD}} \times \frac{\sigma_{\mathrm{NL}}^{\mathrm{EW}}}{\sigma_{\mathrm{LO}}} \equiv \sigma_{\mathrm{NLO}}^{\mathrm{QCD}} \times K_{\mathrm{EW}}
$$

where $\sigma_{\mathrm{NLO}}^{\mathrm{QCD}} \equiv \sigma_{\mathrm{LO}}+\alpha_{s}^{2} \alpha \sigma^{(2,1)}$ and $\sigma_{\mathrm{NLO}}^{\mathrm{EW}} \equiv \sigma_{\mathrm{LO}}+\alpha_{s} \alpha^{2} \sigma^{(1,2)}$. Notice that $\sigma_{\mathrm{NLO}}^{\mathrm{QCD} \times \mathrm{EW}}$ also includes mixed $\mathrm{QCD} \times \mathrm{EW}$ corrections that are of higher order. The second row of Table 1 gives the relative corrections as percentage of the LO cross section, $\delta(\%)=\left(\sigma_{X} / \sigma_{\mathrm{LO}}-1\right) \times 100$ (where X=NLO $\mathrm{QCD}, \mathrm{NLO} \mathrm{EW}, \ldots$... The quoted uncertainty is due to varying the renormalization and factorization scales $\left(\mu=\mu_{r}=\mu_{f}\right)$ between $\mu=M_{Z} / 2$ and $\mu=2 M_{Z}$. The CTEQ14qed PDF set [45] has been used, and no PDF uncertainty has been included in these estimates. If the impact of NLO EW

\begin{tabular}{|c|c|c|c|c|c|}
\hline & LO & NLO QCD & NLO EW & NLO QCD+EW & NLO QCD $\times$ EW \\
\hline \hline$\sigma(\mathrm{pb})$ & $389.73_{-31}^{+15}\left(392.66_{-32}^{+15}\right)$ & $537.7_{-3}^{+7}\left(526.9_{-3}^{+9}\right)$ & $383.40_{-31}^{+15}$ & $531.4_{-3}^{+7}$ & $529.2_{-3}^{+7}$ \\
\hline$\delta(\%)$ & - & $38(34)$ & -1.6 & 36 & 36 \\
\hline \hline
\end{tabular}

Table 1: Total cross sections at LO and NLO (first row) including only QCD NLO corrections, only EW NLO corrections, or both, for $\mu=M_{Z}$ at the LHC with c.m. energy $13 \mathrm{TeV}$. The relative impact of each NLO contribution is given in the second row. The results obtained with $m_{b}=0$ at LO and NLO QCD are given in parenthesis. See text for more details.

corrections on the total cross section is indicative of the average magnitude of their effect, a much more interesting result is their effect on distributions. Furthermore, it is important to compare the effect of NLO EW corrections to the residual theoretical uncertainty of NLO distributions, and estimate in particular whether NLO EW corrections are within the scale uncertainty of the corresponding NLO QCD corrections. This can be quantified in terms of the following ratios:

$$
\delta_{\mathrm{EW}}^{\mathrm{add}}=\frac{\sigma_{\mathrm{NLO}}^{\mathrm{QCD}+\mathrm{EW}}-\sigma_{\mathrm{NLO}}^{\mathrm{QCD}}}{\sigma_{\mathrm{NLO}}^{\mathrm{QCD}}}=\frac{\sigma_{\mathrm{NLO}}^{\mathrm{QCD}+\mathrm{EW}}}{\sigma_{\mathrm{NLO}}^{\mathrm{QCD}}}-1 \quad \text { and } \quad \delta_{\mathrm{EW}}^{\mathrm{prod}}=\frac{\sigma_{\mathrm{NLO}}^{\mathrm{QCD} \times \mathrm{EW}}-\sigma_{\mathrm{NLO}}^{\mathrm{QCD}}}{\sigma_{\mathrm{NLO}}^{\mathrm{QCD}}}=\frac{\sigma_{\mathrm{NLO}}^{\mathrm{EW}}}{\sigma_{\mathrm{LO}}}-1,
$$


for to the additive and multiplicative approach of combining NLO QCD and EW corrections respectively. Following the same logic, we quantify the effect of switching from a massless 5FS to a $\mathrm{m} 5 \mathrm{FS}$ in terms of the ratio

$$
\delta_{\mathrm{mb}}=\frac{\sigma_{\mathrm{NLO}}^{\mathrm{QCD}, \mathrm{mFFS}}-\sigma_{\mathrm{NLO}}^{\mathrm{QCD}, 5 \mathrm{FS}}}{\sigma_{\mathrm{NLO}}^{\mathrm{QCD}, 5 \mathrm{SS}}}=\frac{\sigma_{\mathrm{NLO}}^{\mathrm{QCD}, \mathrm{m} 5 \mathrm{FS}}}{\sigma_{\mathrm{NLO}}^{\mathrm{QCD}, 5 \mathrm{FS}}}-1,
$$

representing the fractional change in the NLO QCD cross sections in going from the 5FS to the m5FS. It is clear that both mass effects and NLO EW corrections are small effects, compared to
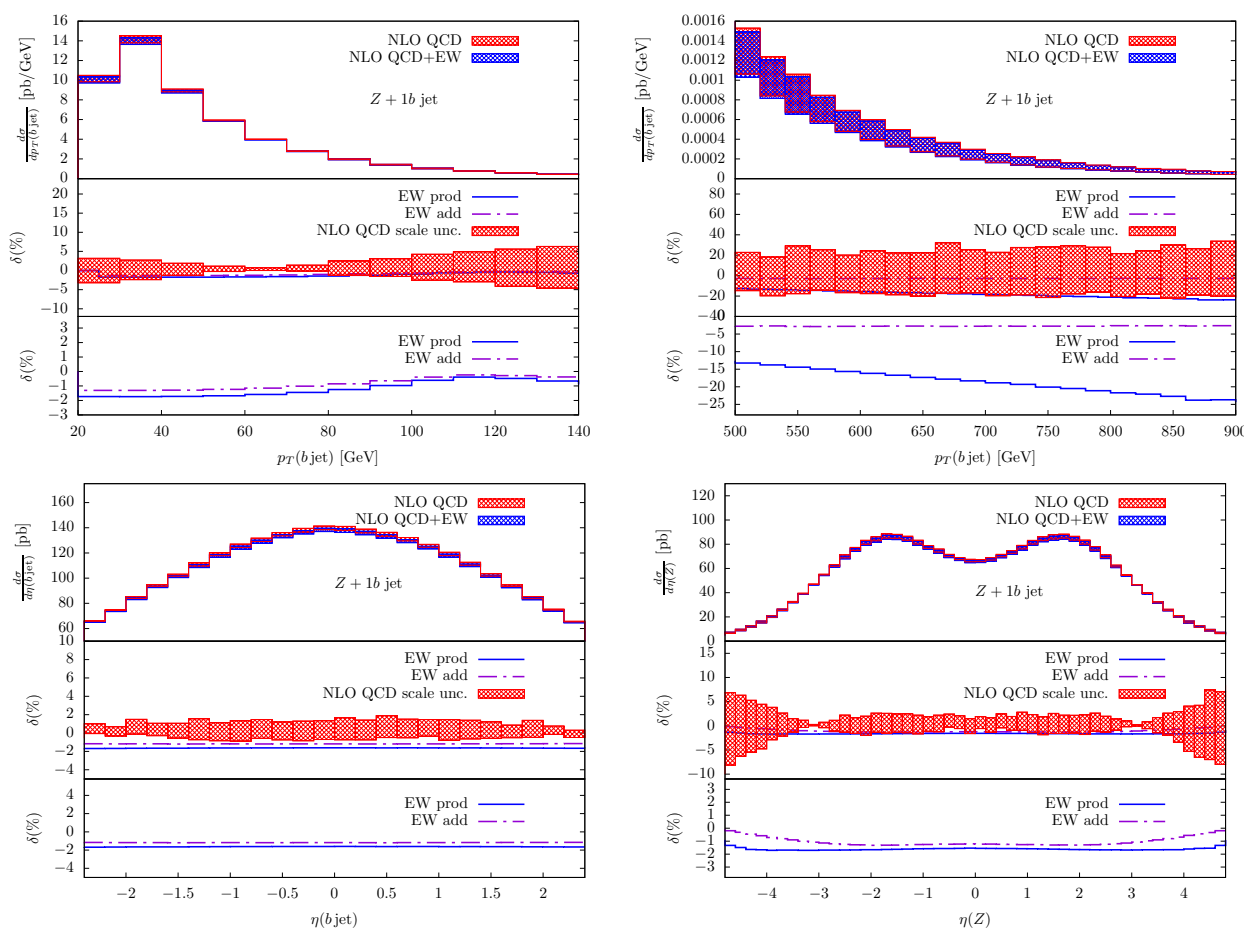

Figure 1: Differential distributions for the $b$-jet transverse momentum, and for the pseudorapidity of both the $b$ jet and $Z$ boson. The lower windows in each plot show the relative EW $O(\alpha)$ corrections differential distributions, $\delta_{\mathrm{EW}}^{\text {prod }}$ and $\delta_{\mathrm{EW}}^{\text {add }}$, together with the NLO QCD scale uncertainty in the middle plot.

the size of NLO QCD corrections, and are mostly within the uncertainty of the NLO QCD cross section. Hence the need for a better control of higher-order QCD corrections. Still, there are clear indications of the impact of EW corrections and mass effects, in particular on angular distributions, and in complementary kinematic regions: EW corrections mainly affect the high- $p_{T}$ region of both $b$-jet and $Z$ boson $p_{T}$, while $b$-quark mass effects are more visible in the low- $p_{T}$ regions. Interestingly, they both tend to be more visible in angular distributions, such as pseudorapidity distributions. One would indeed expect that a modification of the initial-state kinematics can lead to modifications of the final-state angular distributions. This corroborates the original motivation for a consistent implementation of a m5FS in Monte Carlo event generators.

\section{References}

[1] A. Lazopoulos, T. McElmurry, K. Melnikov, and F. Petriello, Next-to-leading order QCD corrections to $t \bar{t} Z$ production at the LHC, Phys. Lett. B666 (2008) 62-65, [arXiv: 0804 . 2220]. 

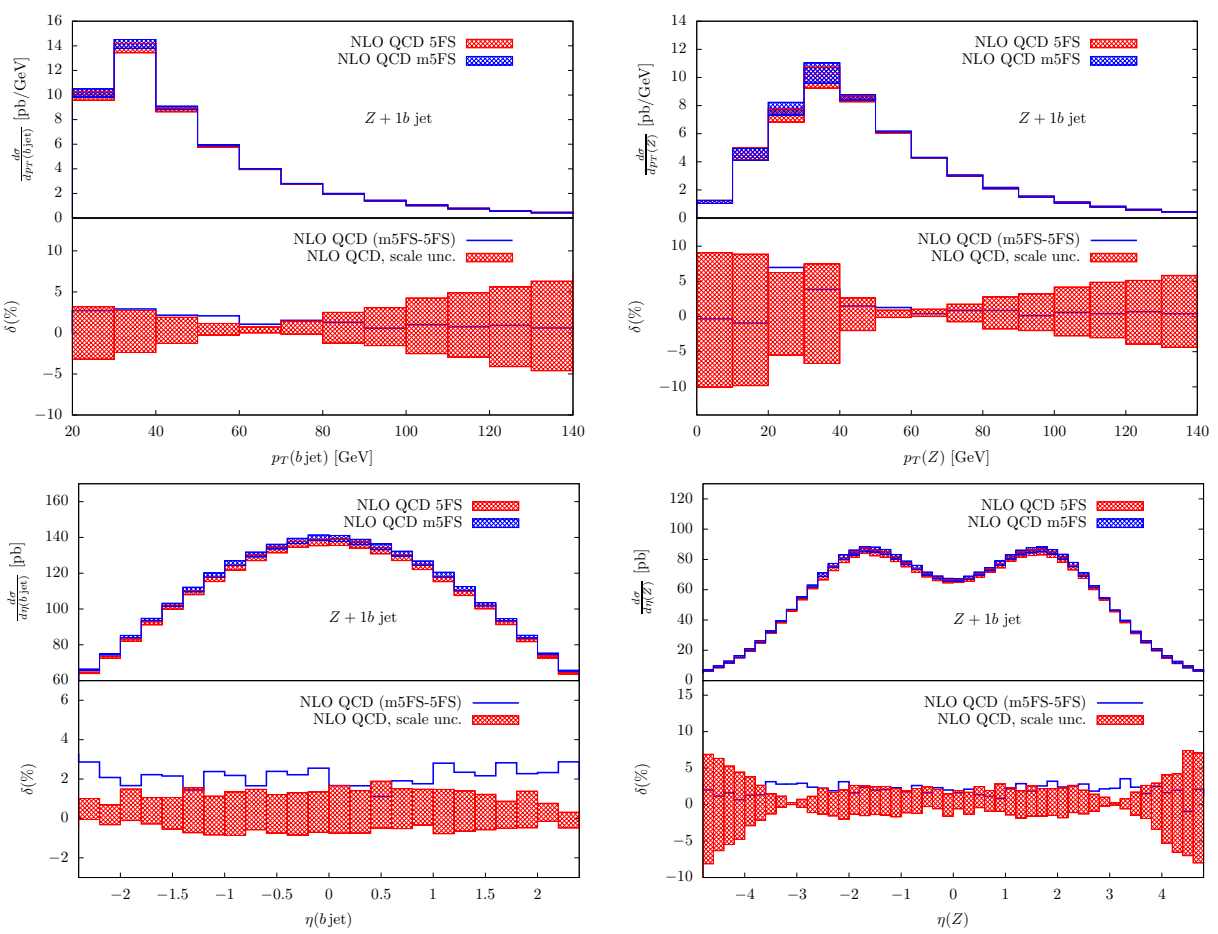

Figure 2: $5 \mathrm{FS}$ and m5FS NLO QCD predictions for differential distributions for the $b$-jet and $Z$ transverse momentum, and the invariant $Z b$-jet mass. The lower window in each plot shows the difference $\delta_{\mathrm{mb}}$ together with the NLO QCD scale uncertainty.

[2] A. Kardos, Z. Trocsanyi, and C. Papadopoulos, Top quark pair production in association with a Z-boson at NLO accuracy, Phys. Rev. D85 (2012) 054015, [arXiv: 1111.0610 ].

[3] J. M. Campbell and R. K. Ellis, $t \bar{t} W^{+-}$production and decay at NLO, JHEP 07 (2012) 052, [arXiv:1204.5678].

[4] M. V. Garzelli, A. Kardos, C. G. Papadopoulos, and Z. Trocsanyi, Z0 - boson production in association with a top anti-top pair at NLO accuracy with parton shower effects, Phys. Rev. D85 (2012) 074022, [arXiv:1111.1444].

[5] M. V. Garzelli, A. Kardos, C. G. Papadopoulos, and Z. Trocsanyi, $t \bar{t} W^{+-}$and $t \bar{t} Z$ Hadroproduction at NLO accuracy in QCD with Parton Shower and Hadronization effects, JHEP 11 (2012) 056, [arXiv:1208.2665].

[6] S. Frixione, V. Hirschi, D. Pagani, H. S. Shao, and M. Zaro, Electroweak and QCD corrections to top-pair hadroproduction in association with heavy bosons, JHEP 06 (2015) 184, [arXiv:1504.03446].

[7] S. Kallweit, J. M. Lindert, P. MaierhÃúfer, S. Pozzorini, and M. SchÃû́nherr, NLO electroweak automation and precise predictions for W+multijet production at the LHC, JHEP 04 (2015) 012, [arXiv:1412.5157].

[8] LHC Higgs Cross Section Working Group Collaboration, D. de Florian et al., Handbook of LHC Higgs Cross Sections: 4. Deciphering the Nature of the Higgs Sector, arXiv:1610.07922.

[9] S. Zhu, Next-to-leading order QCD corrections to bg $\rightarrow$ tW-at CERN large hadron collider, Phys. Lett. B524 (2002) 283-288, [hep-ph/ 01092 69]. [Erratum: Phys. Lett.B537,351(2002)]. 
[10] N. Kidonakis, Two-loop soft anomalous dimensions for single top quark associated production with a W- or H-, Phys. Rev. D82 (2010) 054018, [arXiv: 1005 . 4451].

[11] F. Maltoni, G. Ridolfi, and M. Ubiali, b-initiated processes at the LHC: a reappraisal, JHEP 1207 (2012) 022, [arXiv:1203.6393].

[12] S. Forte, D. Napoletano, and M. Ubiali, Higgs production in bottom-quark fusion in a matched scheme, Phys. Lett. B751 (2015) 331-337, [arXiv: 1508.01529 ].

[13] F. Febres Cordero and L. Reina, Electroweak Gauge-Boson Production in Association with b Jets at Hadron Colliders, Int. J. Mod. Phys. A30 (2015), no. 16 1530042, [arXiv: 1504.07177 ].

[14] M. Lim, F. Maltoni, G. Ridolfi, and M. Ubiali, Anatomy of double heavy-quark initiated processes, JHEP 09 (2016) 132, [arXiv: 1605.09411$].$

[15] S. Forte, D. Napoletano, and M. Ubiali, Higgs production in bottom-quark fusion: matching beyond leading order, Phys. Lett. B763 (2016) 190-196, [arXiv: 1607.0038 9].

[16] M. Bonvini, A. S. Papanastasiou, and F. J. Tackmann, Resummation and matching of b-quark mass effects in $b \bar{b} H$ production, JHEP 11 (2015) 196, [arXiv: 1508 .03288].

[17] M. Bonvini, A. S. Papanastasiou, and F. J. Tackmann, Matched predictions for the $b \bar{b} H$ cross section at the 13 TeV LHC, JHEP 10 (2016) 053, [arXiv:1605.01733].

[18] F. Krauss, D. Napoletano, and S. Schumann, Simulating b-associated production of Z and Higgs bosons with the SHERPA event generator, Phys. Rev. D95 (2017), no. 3 036012, [arXiv:1612.04640].

[19] J. M. Campbell, R. K. Ellis, F. Maltoni, and S. Willenbrock, Production of a W boson and two jets with one $b^{-}$quark tag, Phys.Rev. D75 (2007) 054015, [hep-ph/ 0611348$]$.

[20] J. M. Campbell, R. K. Ellis, F. Febres Cordero, F. Maltoni, L. Reina, et al., Associated Production of a W Boson and One b Jet, Phys.Rev. D79 (2009) 034023, [arXiv: 0809.3003 ].

[21] J. Campbell, F. Caola, F. Febres Cordero, L. Reina, and D. Wackeroth, NLO QCD predictions for $W+1$ jet and $W+2$ jet production with at least one b jet at the 7 TeV LHC, Phys.Rev. D86 (2012) 034021, [arXiv:1107.3714].

[22] F. Febres Cordero, L. Reina, and D. Wackeroth, NLO QCD corrections to W boson production with a massive b-quark jet pair at the Tevatron p anti-p collider, Phys.Rev. D74 (2006) 034007, [hep-ph/0606102].

[23] F. Febres Cordero, L. Reina, and D. Wackeroth, W- and Z-boson production with a massive bottom-quark pair at the Large Hadron Collider, Phys.Rev. D80 (2009) 034015, [arXiv:0906.1923].

[24] S. Badger, J. M. Campbell, and R. Ellis, QCD corrections to the hadronic production of a heavy quark pair and a W-boson including decay correlations, JHEP 1103 (2011) 027, [arXiv: 1011.6647 ].

[25] G. Luisoni, C. Oleari, and F. Tramontano, Wbbj production at NLO with POWHEG+MiNLO, arXiv:1502.01213.

[26] L. Reina and T. Schutzmeier, Towards Wb bbar + j at NLO with an Automatized Approach to One-Loop Computations, JHEP 1209 (2012) 119, [arXiv: 1110 . 4438].

[27] J. M. Campbell, R. K. Ellis, F. Maltoni, and S. Willenbrock, Associated production of a Z Boson and a single heavy quark jet, Phys.Rev. D69 (2004) 074021, [hep-ph / 0312024 ]. 
[28] J. M. Campbell, R. K. Ellis, F. Maltoni, and S. Willenbrock, Production of a Z boson and two jets with one heavy-quark tag, Phys.Rev. D73 (2006) 054007, [hep-ph / 0510362 ].

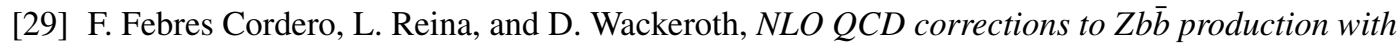
massive bottom quarks at the Fermilab Tevatron, Phys.Rev. D78 (2008) 074014, [arXiv:0806.0808].

[30] F. R. Anger, F. Febres Cordero, H. Ita, and V. Sotnikov, NLO QCD predictions for Wb $\bar{b}$ production in association with up to three light jets at the LHC, Phys. Rev. D97 (2018), no. 3036018 , [arXiv:1712.05721].

[31] T. Sjostrand, S. Mrenna, and P. Z. Skands, PYTHIA 6.4 Physics and Manual, JHEP 0605 (2006) 026, [hep-ph/0603175].

[32] T. Sjostrand, S. Mrenna, and P. Z. Skands, A Brief Introduction to PYTHIA 8.1, Comput.Phys.Commun. 178 (2008) 852-867, [arXiv: 0710.3820 ].

[33] G. Marchesini, B. Webber, G. Abbiendi, I. Knowles, M. Seymour, et al., HERWIG: A Monte Carlo event generator for simulating hadron emission reactions with interfering gluons. Version 5.1 - April 1991, Comput.Phys.Commun. 67 (1992) 465-508.

[34] G. Corcella, I. Knowles, G. Marchesini, S. Moretti, K. Odagiri, et al., HERWIG 6: An Event generator for hadron emission reactions with interfering gluons (including supersymmetric processes), JHEP 0101 (2001) 010, [hep-ph / 0011363 ].

[35] S. Alioli, P. Nason, C. Oleari, and E. Re, A general framework for implementing NLO calculations in shower Monte Carlo programs: the POWHEG BOX, JHEP 1006 (2010) 043, [arXiv : 1002.2581 ].

[36] C. Oleari and L. Reina, $W+-b \bar{b}$ production in POWHEG, JHEP 1108 (2011) 061, [arXiv:1105.4488].

[37] J. Alwall, R. Frederix, S. Frixione, V. Hirschi, F. Maltoni, et al., The automated computation of tree-level and next-to-leading order differential cross sections, and their matching to parton shower simulations, JHEP 1407 (2014) 079, [arXiv: 1405.0301 ].

[38] R. Frederix, S. Frixione, V. Hirschi, F. Maltoni, R. Pittau, et al., $W$ and $Z / \gamma *$ boson production in association with a bottom-antibottom pair, JHEP 1109 (2011) 061, [arXiv: 1106.6019 ].

[39] J. R. Andersen et al., Les Houches 2015: Physics at TeV Colliders Standard Model Working Group Report, in 9th Les Houches Workshop on Physics at TeV Colliders (PhysTeV 2015) Les Houches, France, June 1-19, 2015, 2016. arXiv:1605.04692.

[40] J. M. Campbell, R. K. Ellis, and C. Williams, Mcfm, from v.8.0, 2015. http: / /mcfm. fnal.gov.

[41] D. Figueroa, S. Honeywell, S. Quackenbush, L. Reina, C. Reuschle, and D. Wackeroth, Electroweak and QCD corrections to Z-boson production with one b jet in a massive 5 Flavor Scheme, arXiv: 1805.01353.

[42] J. C. Collins, Hard scattering factorization with heavy quarks: A General treatment, Phys.Rev. D58 (1998) 094002, [hep-ph/9806259].

[43] F. Krauss and D. Napoletano, Towards a fully massive five-flavour scheme, arXiv:1712.06832.

[44] S. Honeywell, S. Quackenbush, L. Reina, and C. Reuschle, NLOX release note and manual, NLOX release note and manual (in preparation).

[45] C. Schmidt, J. Pumplin, D. Stump, and C. P. Yuan, CT14QED parton distribution functions from isolated photon production in deep inelastic scattering, Phys. Rev. D93 (2016), no. 11 114015, [arXiv:1509.02905]. 